Supporting Information:

\title{
Selective Self-Assembly of a Rectangular Ruthenium Supramolecule from an Unsymmetrical Bridging Unit
}

Eun Hye Wi†, Ji Yeon Ryư ${ }^{\dagger}$, Seul Gi Lee, Ume Farwa, Moumita Pait, Sunwoo Lee, Sung Cho, and Junseong Lee*

Department of Chemistry, Chonnam National University, 77 Yongbong-ro, Buk-gu, Gwangju 61186, Republic of Korea. Fax: 8262530 3389; Tel: 82625303371 ;

E-mail: leespy@chonnam.ac.kr

Table of Contents:

1. IR and NMR spectra of complex 1, 2 and 3

2. Mass spectra of complex 2

3. X-ray structure determination 
1. IR and NMR spectra of complex 1, 2 and 3

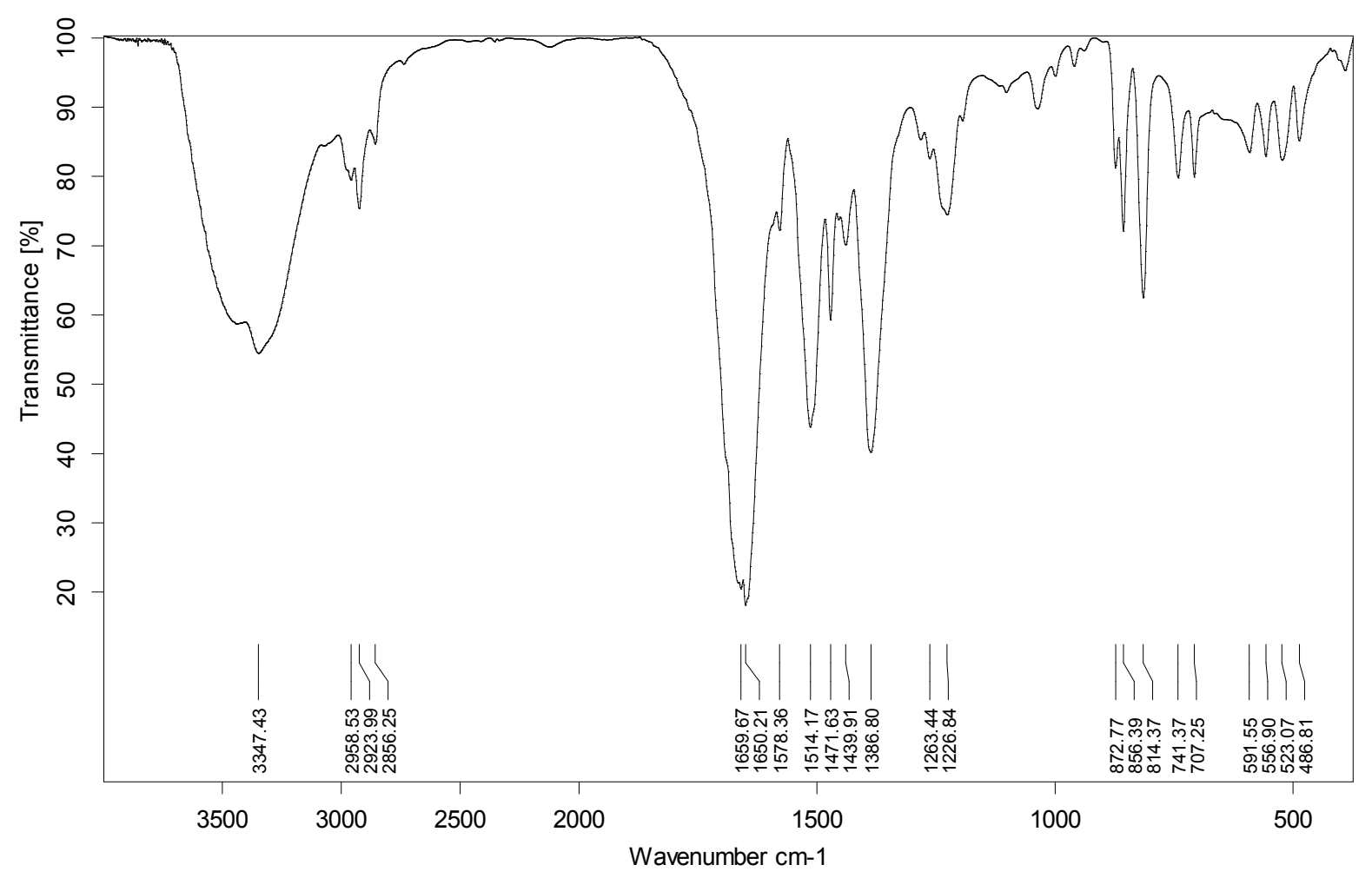

Figure S1. IR Spectrum of 1. 


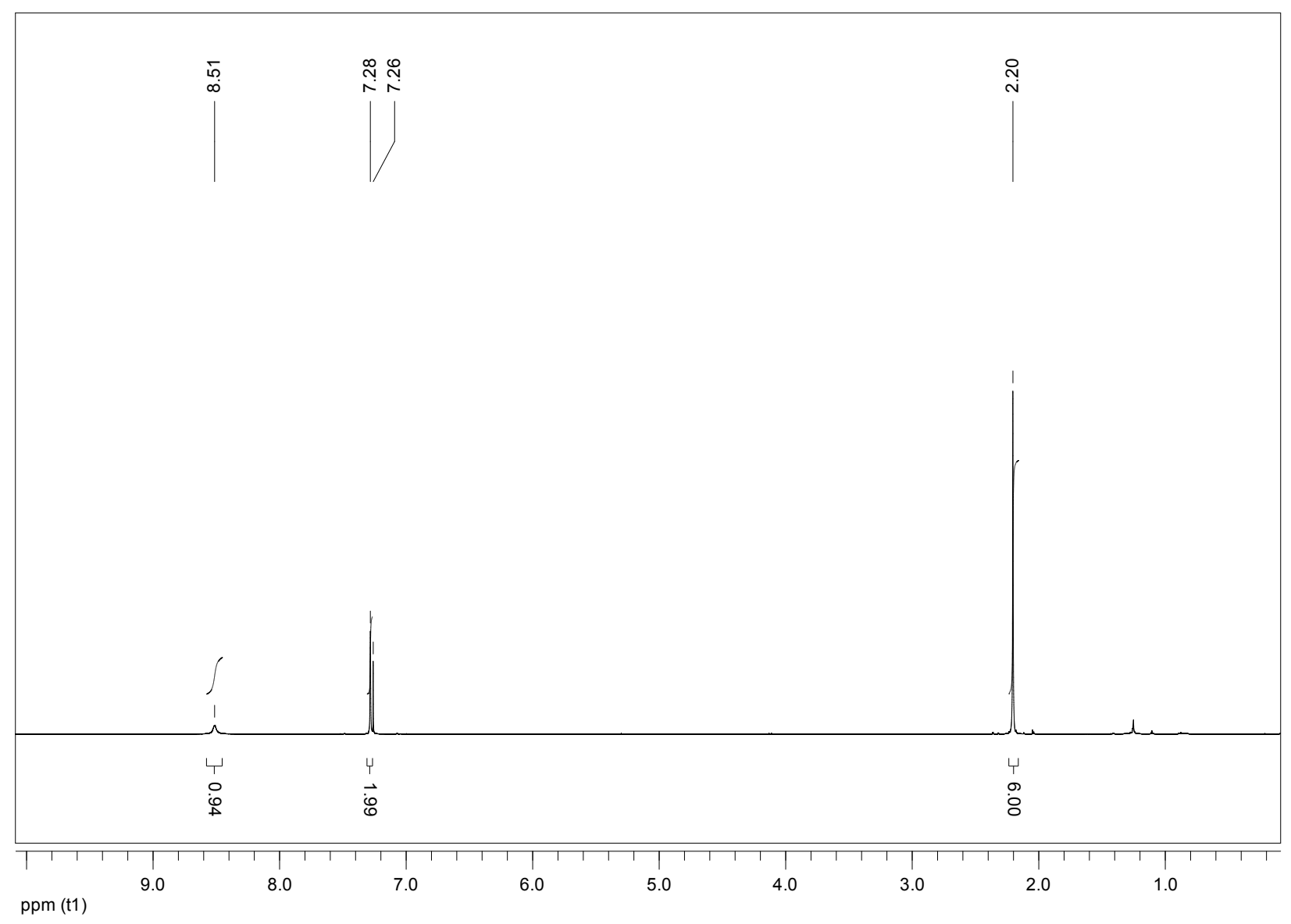

Figure S2. ${ }^{1} \mathrm{H}$ NMR spectrum of 1.

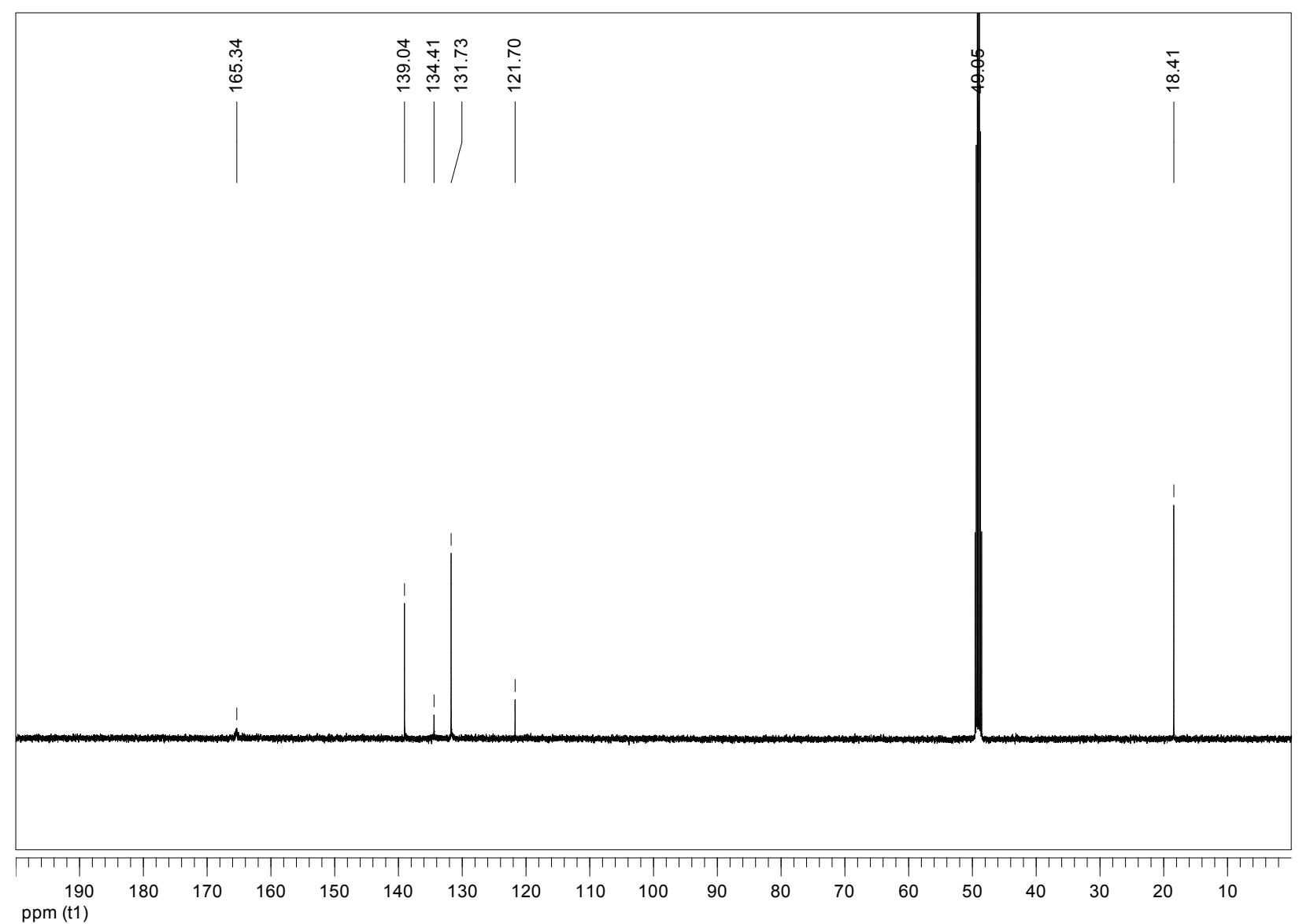


Figure S3. ${ }^{13} \mathrm{C}$ NMR spectrum of 1.

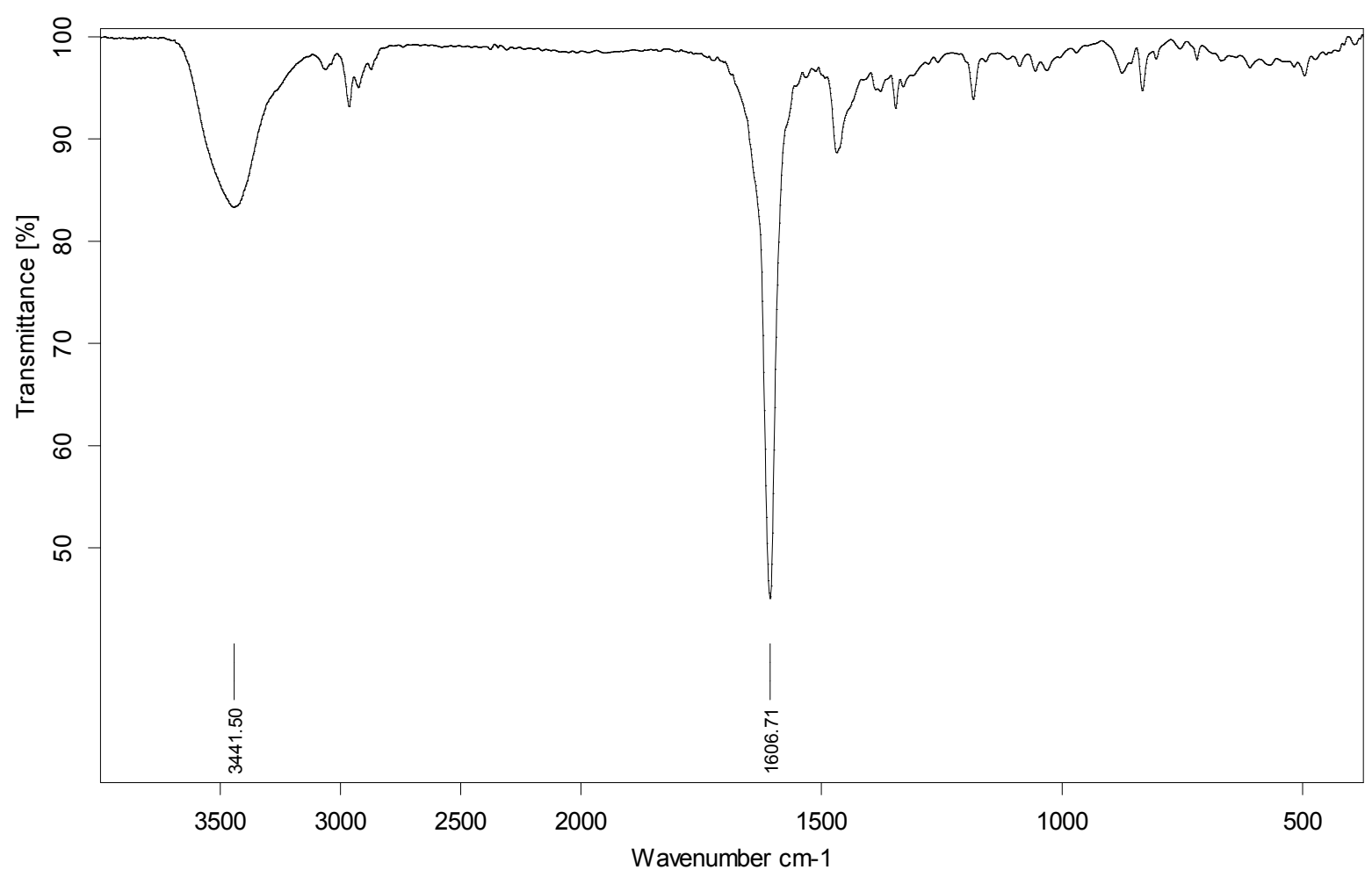

Figure S4. IR Spectrum of 2.

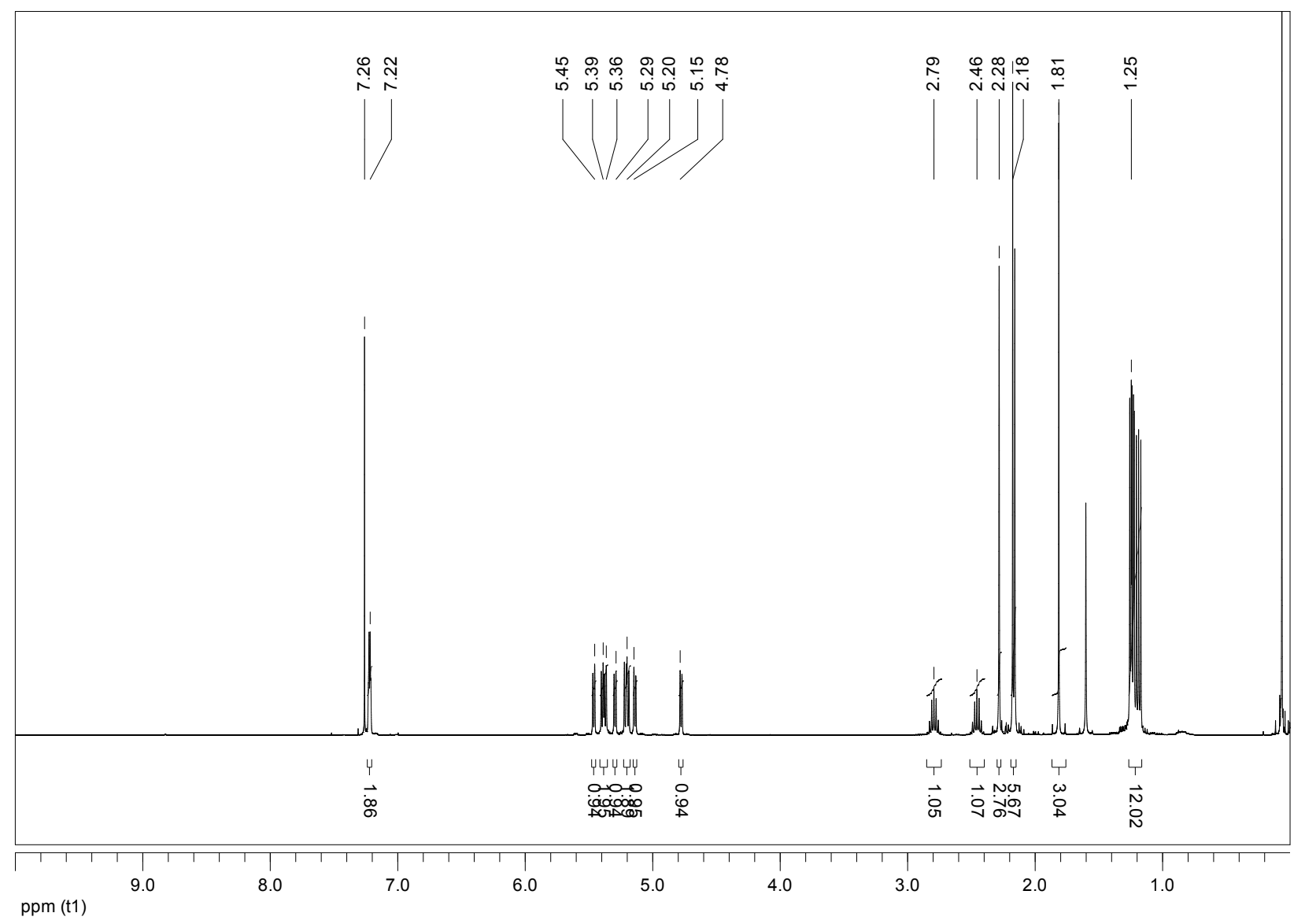


Figure S5. ${ }^{1} \mathrm{H}$ NMR spectrum of 2.

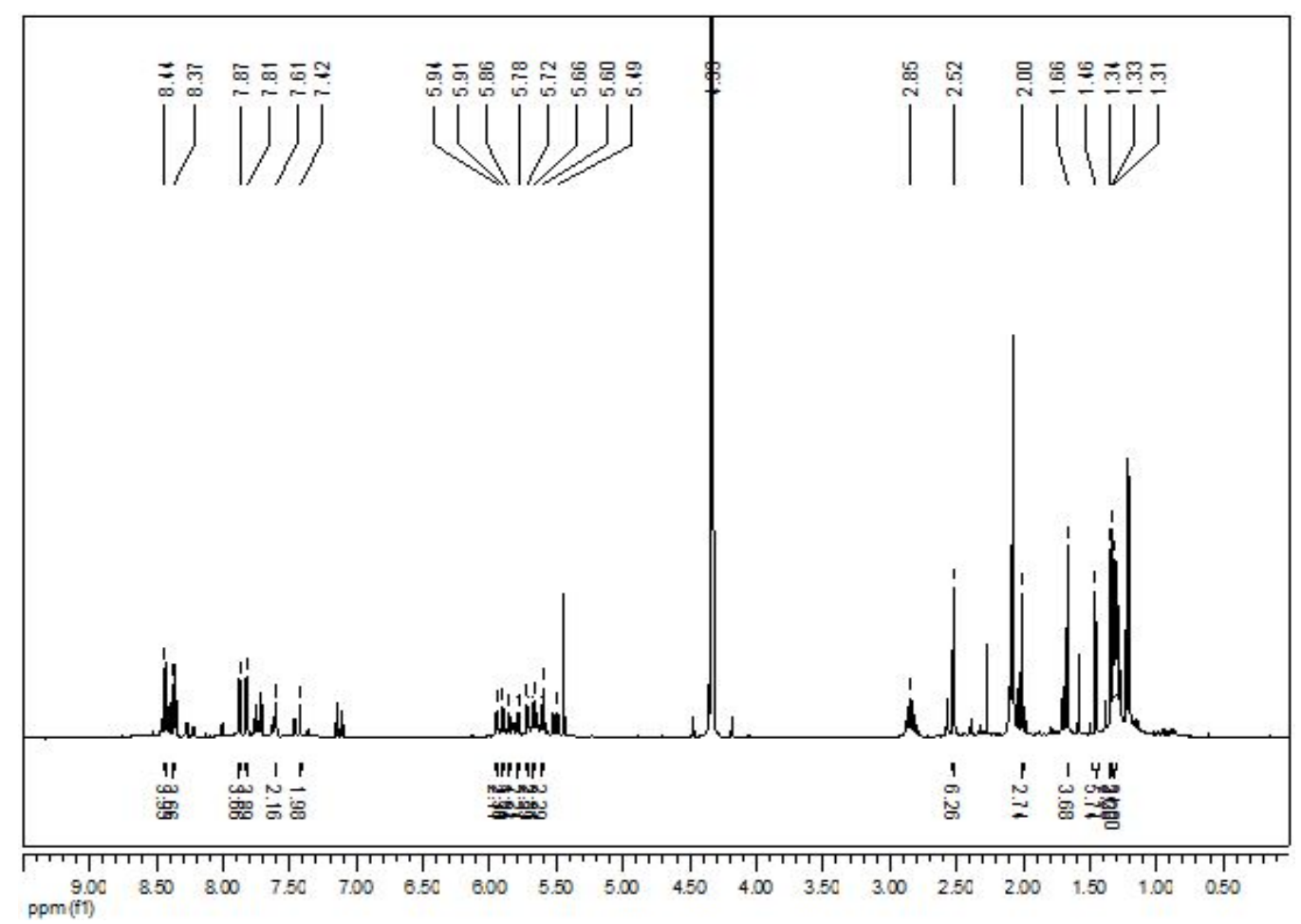

Figure S6. ${ }^{1} \mathrm{H}$ NMR spectrum of 3.

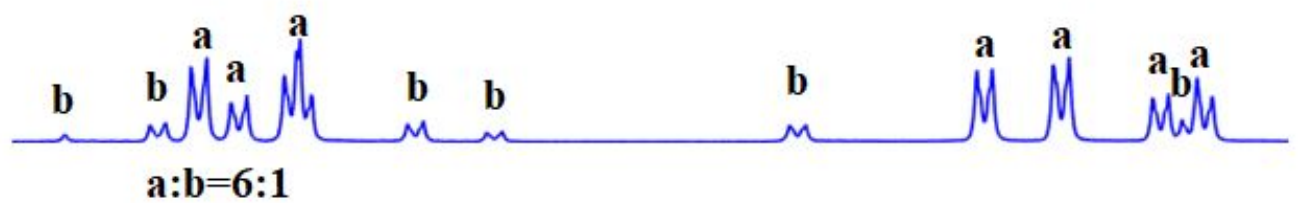

Figure S7. Partial ${ }^{1} \mathrm{H}$ NMR spectrum of 3 in aromatic region. 


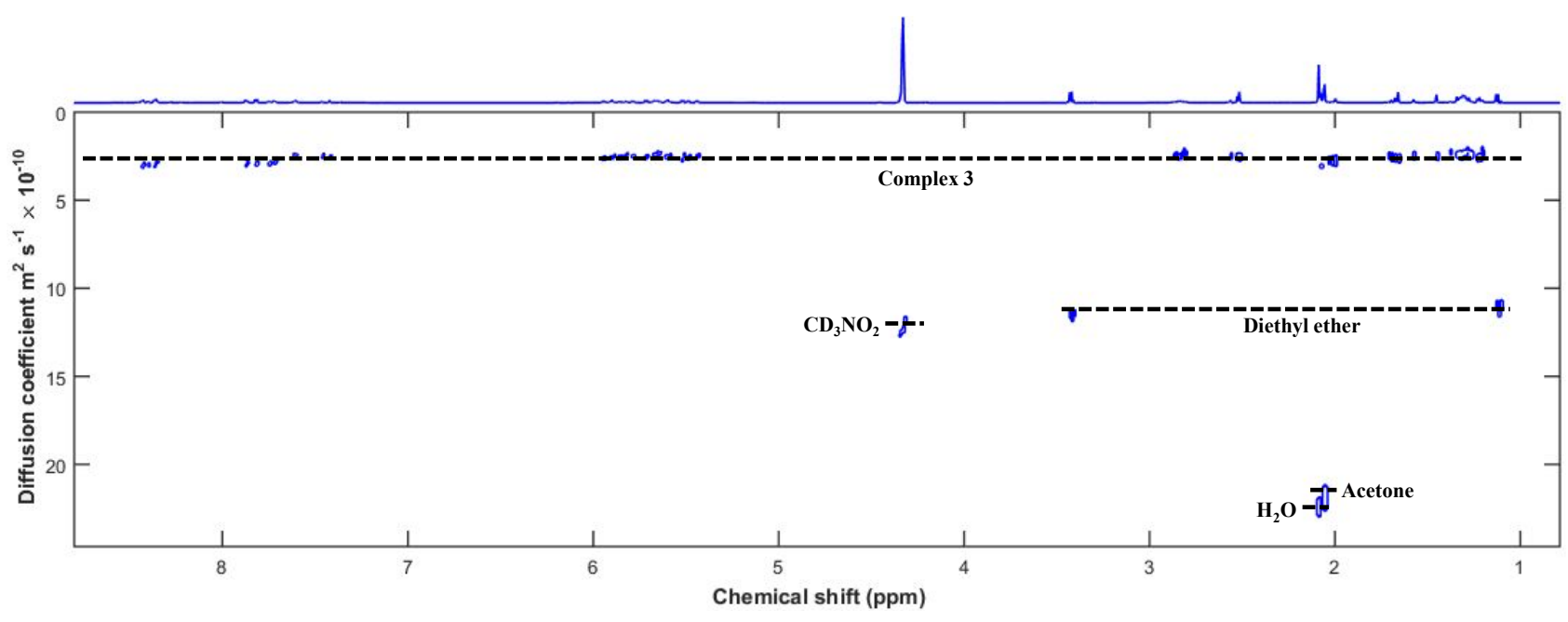

Figure S8. DOSY NMR spectrum of 3. 
2. Mass spectra of complex 2

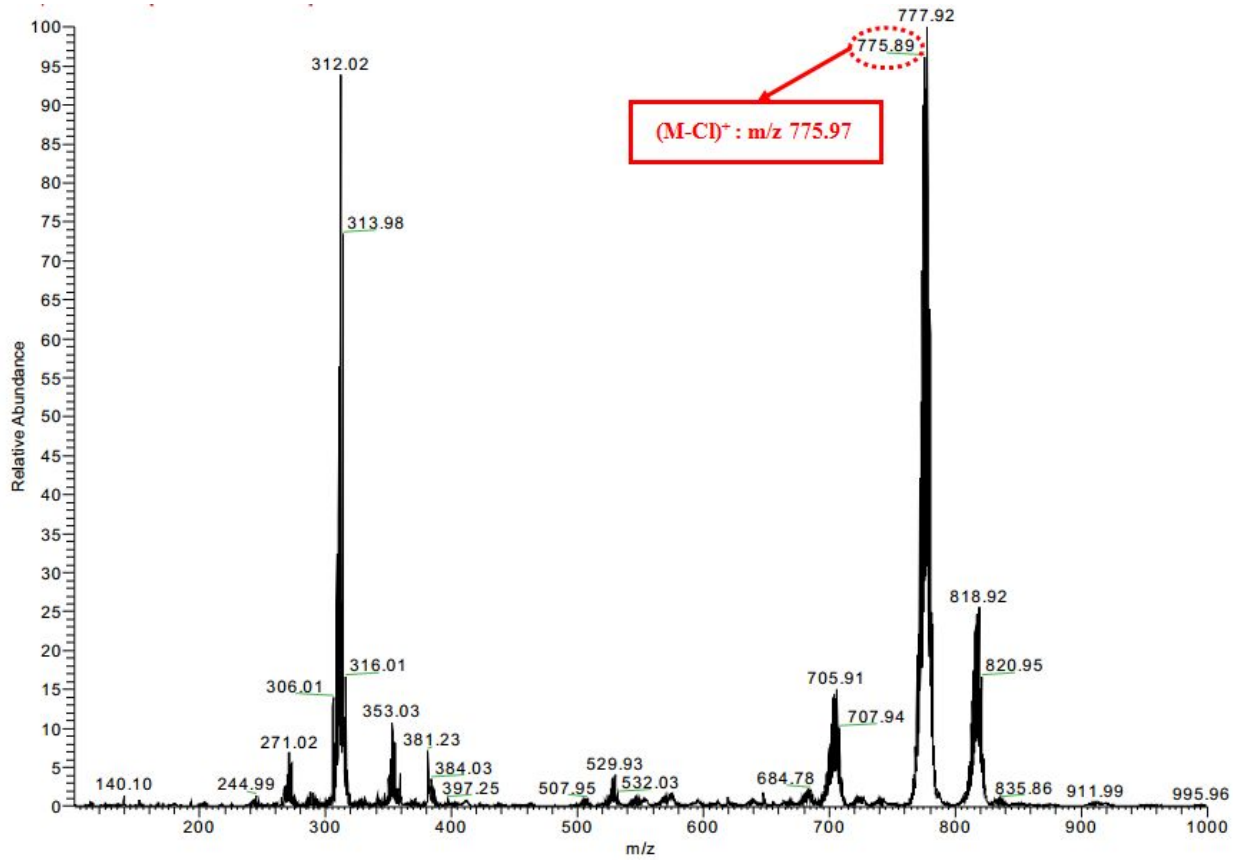

Figure S9. Full ESI-TQ-MS spectrum of 2. 

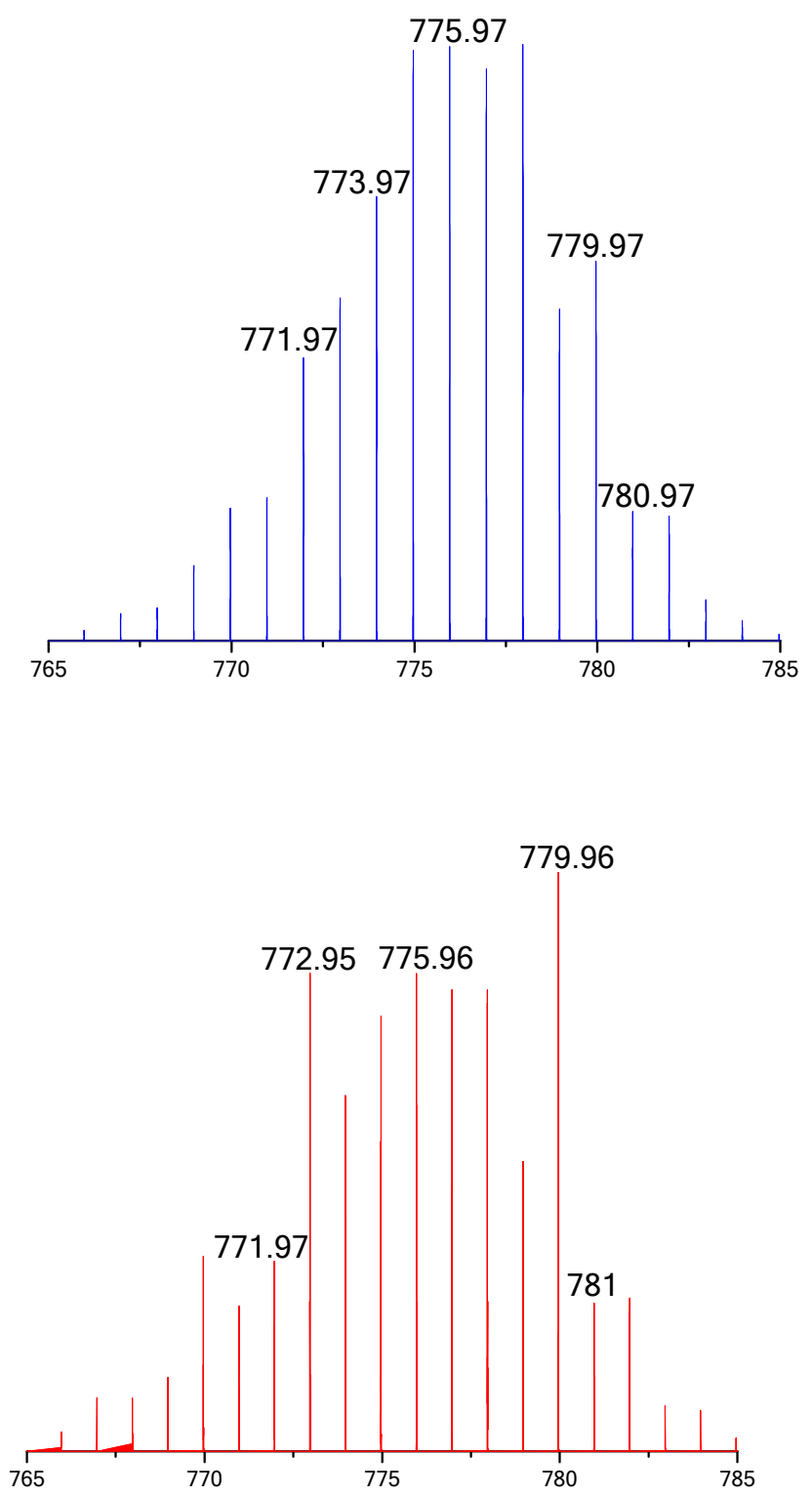

Figure 10. Calculated(blue) and Experimental(red) ESI-TQ-MS of diruthenium complex 2. 


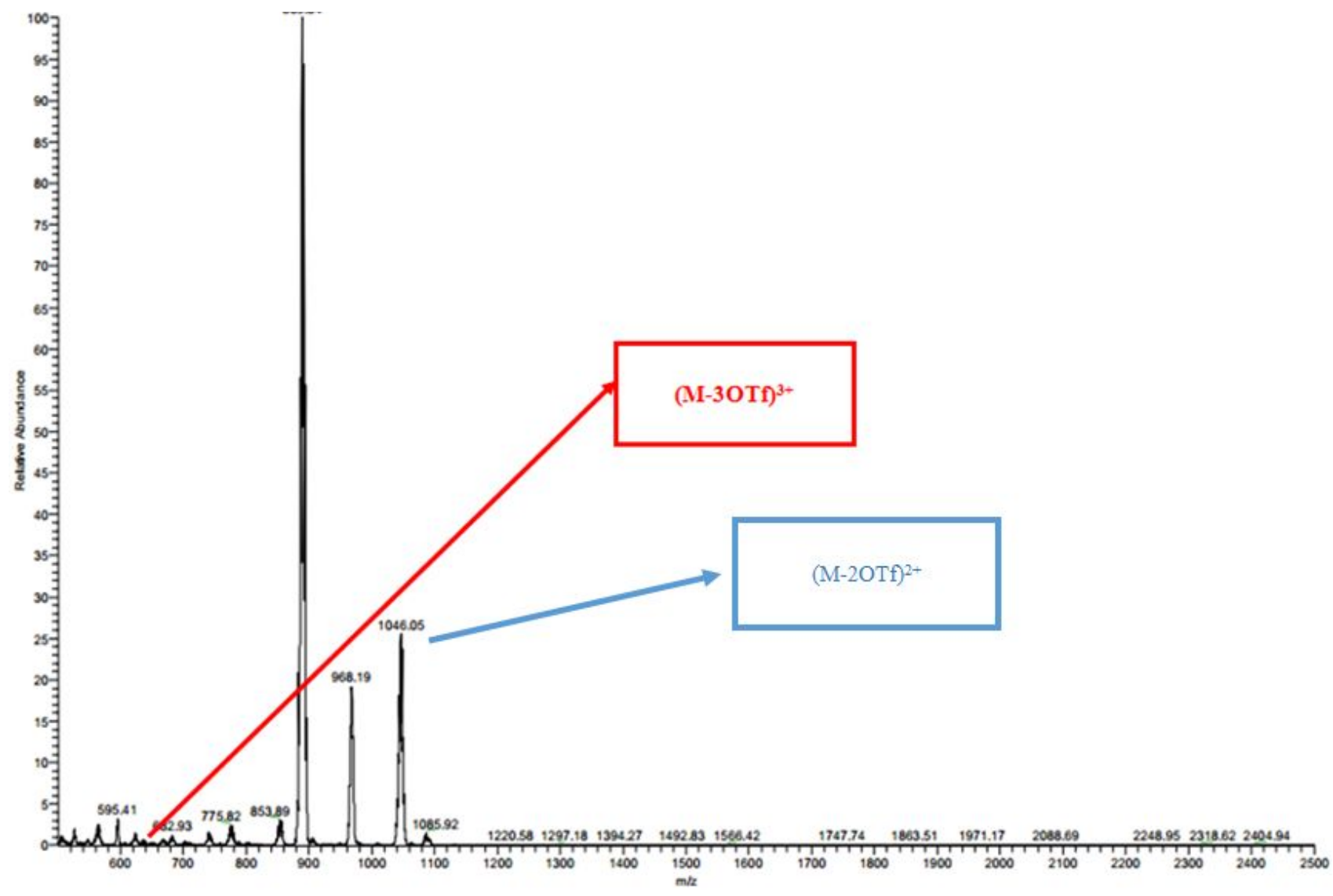

Figure S11. Full ESI-TQ-MS spectrum of supramolecule 3. 


\section{X-ray structure determination}

Table S1. Crystallographic data and parameters for 2 and 3.

\begin{tabular}{|c|c|c|}
\hline & 2 & 3 \\
\hline formula & $\mathrm{C}_{32} \mathrm{H}_{37.50} \mathrm{BrCl}_{2} \mathrm{NO}_{4} \mathrm{Ru}_{2} \mathrm{~S}$ & $\mathrm{C}_{93} \mathrm{Br}_{2} \mathrm{FN}_{6} \mathrm{O}_{8} \mathrm{Ru}_{4} \mathrm{~S}$ \\
\hline formula weight & 885.14 & 1944.15 \\
\hline crystal system & Triclinic & Triclinic \\
\hline space group & $P-1$ & $P-1$ \\
\hline$a(\AA)$ & $8.6701(10)$ & $13.1050(7)$ \\
\hline$b(\AA)$ & $20.376(2)$ & $18.7851(11)$ \\
\hline$c(\AA)$ & $20.959(2)$ & $23.4278(13)$ \\
\hline$\alpha\left(^{\circ}\right)$ & $70.432(7)$ & $89.891(3)$ \\
\hline$\beta\left(^{\circ}\right)$ & $89.048(8)$ & 73.764(3) \\
\hline$\gamma\left({ }^{\circ}\right)$ & $85.058(7)$ & $89.976(3)$ \\
\hline$V\left(\AA^{3}\right)$ & $3475.5(7)$ & $5537.4(5)$ \\
\hline$Z$ & 4 & 2 \\
\hline$\rho_{\text {calc }}\left(\mathrm{g} \mathrm{cm}^{-3}\right)$ & 1.692 & 1.166 \\
\hline$\mu\left(\mathrm{mm}^{-1}\right)$ & 2.268 & 1.322 \\
\hline$F(000)$ & 1766 & 1870 \\
\hline$T(\mathrm{~K})$ & $100(2)$ & $100(2)$ \\
\hline scan mode & $\omega$ and $\phi$ & $\omega$ and $\phi$ \\
\hline$h k /$ range & $\begin{array}{l}-7<=\mathrm{h}<=7 \\
-16<=\mathrm{k}<=17 \\
-16<=1<=17\end{array}$ & $\begin{array}{l}-13<=\mathrm{h}<=13, \\
-18<=\mathrm{k}<=19 \\
-24<=1<=23\end{array}$ \\
\hline measd reflns & 8381 & 29727 \\
\hline unique reflns $\left[R_{\text {int }}\right]$ & 4162 & 12356 \\
\hline refined parameters & 742 & 836 \\
\hline $\mathrm{R}_{1}{ }^{a}(\mathrm{I}>2 \sigma(\mathrm{I}))$ & 0.1263 & 0.2916 \\
\hline $\mathrm{wR}_{2}{ }^{b}$ all data & 0.3347 & 0.6713 \\
\hline GOF on $F^{2}$ & 1.083 & 2.384 \\
\hline
\end{tabular}


${ }^{a} \mathrm{R} 1=\Sigma|| F_{\mathrm{O}}|-| F \mathrm{C}|| \Sigma\left|F_{\mathrm{O}}\right| .{ }^{b} \mathrm{WR} 2=\left\{\left[\sum W\left(F_{\mathrm{O}}{ }^{2}-F_{\mathrm{C}}{ }^{2}\right)^{2}\right] /\left[\sum W\left(F^{2}\right)^{2}\right]\right\}^{1 / 2}$. 